Revista

Ibero-Americana

de Estratégıa
RIAE - Revista Ibero-Americana de Estratégia

\title{
VISÃO BASEADA EM RECURSOS DINÂMICOS: ESTUDO DAS CONTRIBUIÇÕES DA ÁREA DE DINÂMICA DE SISTEMAS (DS) PARA A TEORIA DA VISÃO BASEADA EM RECURSOS (VBR)
}

\author{
DYNAMIC RESOURCE BASED-VIEW: CONTRIBUITIONS OF THE SYSTEM \\ DYNAMICS (DS) FOR THE RESOURCE BASED VIEW THEORY (VBR)
}

VISIÓN BASADA EN LOS RECURSOS DINÁMICOS: ESTUDIO DE LAS CONTRIBUCIONES DEL ÁREA DE DINÁMICA DE SISTEMAS (DS) PARA LA TEORÍA DE LA VISIÓN BASADA EN LOS RECURSOS (VBR)

\section{Josué Vitor de Medeiros Júnior}

Professor do curso de Sistemas de Informação na Universidade Federal do Rio Grande de Norte UFRN - SP, Brasil

E-mail: josuevitor@yahoo.com.br

\section{Miguel Eduardo Moreno Añez}

Professor do Programa de Pós-Graduação em Administração da Universidade Federal do Rio Grande do Norte - PPGA/UFRN - RN, Brasil

E-mail: anez1957@yahoo.com.br

Isabella Freitas Gouveia de Vasconcelos

Professora do Stricto Sensu em Administração da Fundação Educacional Inaciana Padre Saboia de Medeiros - FEI - SP, Brasil

E-mail: ivasconcelos@yahoo.com

Fernando Porfírio Soares de Oliveira

Professor do Núcleo de Educação Tecnológica da Faculdade de Ciências, Cultura e Extensão FACEX - RN, Brasil

E-mail: fernandoporfiri@uol.com.br 
Josué Vitor de Medeiros Júnior, Miguel Eduardo Moreno Añez, Isabella Freitas G. de Vasconcelos e Fernando Porfírio S. de Oliveira

\section{VISÃO BASEADA EM RECURSOS DINÂMICOS: ESTUDO DAS CONTRIBUIÇÕES DA ÁREA DE DINÂMICA DE SISTEMAS (DS) PARA A TEORIA DA VISÃO BASEADA EM RECURSOS (VBR)}

\section{RESUMO}

O presente trabalho objetiva avaliar as contribuições conceituais da área de Dinâmica de Sistemas (DS) para a Teoria da Visão Baseada em Recursos (VBR), um campo de estudo da área de Estratégia. Para tanto, realizou-se um levantamento bibliográfico nos últimos anos na área de Estratégia e que contribuições a área de DS pode trazer neste contexto. Especificamente, é apresentada a arquitetura estratégica proposta por Warren (2002) que se utiliza dos dois conceitos para possibilitar análise das dependências entre os recursos e seus impactos dinâmicos no desempenho organizacional. Como resultado, pôde-se perceber que alguns aspectos não abordados pela VBR podem receber contribuições importantes provenientes da DS, como a oportunidade de construir modelos estratégicos que possibilitem a simulação, permitindo a análise da interdependência entre os recursos tangíveis e intangíveis, considerando não apenas o desempenho da empresa em um ponto no tempo, mas sua trajetória temporal e dinâmica. Pretende-se a partir deste trabalho, estender os estudos para aplicações dos seus resultados na área de ensino de estratégia, através de simuladores educacionais construídos com base nas duas áreas.

Palavras-chave: Dinâmica de sistemas; Visão baseada em recursos; Dynamic resource based-view; Estratégia.

\section{DYNAMIC RESOURCE BASED-VIEW: CONTRIBUITIONS OF THE SYSTEM DYNAMICS (DS) FOR THE RESOURCE BASED VIEW THEORY (VBR)}

\section{ABSTRACT}

This work aims to evaluate the conceptual contributions from the area of System Dynamics (SD) to the Resource Based-View (RBV) Theory, a field of study in the Strategy area. With that aim in mind, a literature review of the Strategy field in the latest years was carried out and the contributions that SD can offer in this context were analyzed. Specifically, the strategic architecture proposed by Warren (2002) is presented: it employs the two concepts to make the analysis of dependencies between resources and their dynamic impacts on organizational performance possible. As a result, it was observed that some aspects that are not addressed by the RBV theory can receive important contributions from SD, such as the opportunity to build strategic models that permit simulation, making the analysis of the interdependence between tangible and intangible resources possible, considering not only the performance of the company at a point in time, but its temporal trajectory and dynamics. This work, therefore, proposes to extend the studies on the applications of its results in the Strategy teaching area, through the use of educational simulators which are based on those two 
Visão Baseada em Recursos Dinâmicos: Estudo das Contribuições da Área de Dinâmica de Sistemas (DS) para a Teoria da Visão Baseada em Recursos (VBR)

Keywords: Resource based view; Strategy; System dynamics.

VISIÓN BASADA EN LOS RECURSOS DINÁMICOS: ESTUDIO DE LAS

CONTRIBUCIONES DEL ÁREA DE DINÁMICA DE SISTEMAS (DS) PARA LA TEORÍA

DE LA VISIÓN BASADA EN LOS RECURSOS (VBR)

\section{RESUMO}

El presente trabajo tiene como objetivo evaluar las contribuciones conceptuales del área de Dinámica de Sistemas (DS) para la Teoría de la Visión Basada en los Recursos (VBR), un campo de estudio del área de Estrategia. Para ello, se realizó un levantamiento bibliográfico de los últimos años en el área de Estrategia y que contribuciones el área de DS puede traer en este contexto. Específicamente, se presenta la arquitectura estratégica propuesta por Warren (2002) que utiliza los dos conceptos para posibilitar el análisis de las dependencias entre los recursos y sus impactos dinámicos en el desempeño organizacional. Como resultado, se puede percibir que algunos aspectos no abordados por la VBR pueden recibir contribuciones importantes provenientes de la DS, como la oportunidad de construir modelos estratégicos que posibiliten la simulación, permitiendo el análisis de la interdependencia entre los recursos tangibles e intangibles, considerando no apenas el desempeño de la empresa en un punto en el tiempo, sino su trayectoria temporal y dinámica. A partir de este trabajo, se pretende extender los estudios para aplicaciones de sus resultados en el área de la enseñanza de estrategia, a través de simuladores educacionales construidos con base en las dos áreas.

Palabras clave: Dinámica de sistemas; Visión basada en los recursos; Dynamic resource based view; Estrategia. 
Josué Vitor de Medeiros Júnior, Miguel Eduardo Moreno Añez, Isabella Freitas G. de Vasconcelos e Fernando Porfírio S. de Oliveira

\section{INTRODUÇÃO}

A área de estratégia nas organizações é influenciada por diversas correntes de pensamentos e variadas ênfases e direcionamentos. Mintzberg, Ahlstrand e Lampel (2000) apresentam 10 escolas que influenciam o pensamento estratégico, demonstrando o quanto algumas permanecem contemporâneas.

Especificamente, as teorias da estratégia organizacional que tratam de questões relacionadas à vantagem competitiva podem ter duas ênfases principais (Vasconcelos \& Cyrino, 2000): uma que contextualiza a vantagem como um atributo de posicionamento, e portanto exterior à organização e relacionada à dinâmica do mercado; e uma segunda que enfatiza características internas como principais responsáveis pelo desempenho superior e portanto vantagem competitiva das organizações.

Nesse contexto, e pertencente à segunda ênfase, a Visão Baseada em Recursos, ou Resource Based View (RBV), é uma teoria relacionada à explicação da vantagem competitiva sustentável de uma organização e seu desempenho, a partir de seus recursos estratégicos, como ativos, competências e habilidades. Fleury e Fleury (2003) descrevem que esta teoria é mais adequada às constantes mudanças ambientais complexas do ambiente ao qual as organizações se encontram e descrevem que a abordagem propiciada pela RBV de dentro para fora é mais adequada para se compreender como as estratégias são concebidas e executadas.

Schneider e Lieb (2004 citado por Sheehan, 2006) argumentam que apesar da importância da teoria do RBV, ela é pouco representativa no ensino da estratégia devido a alguns fatores, como a dominância da visão econômica industrial nas instituições (e notadamente a escola do posicionamento como conteúdo obrigatório representa esta influência) e a ausência de um modelo que simplifique o entendimento da RBV.

Além disso, críticas à própria natureza da teoria ressaltam que esta não se preocupa com a compreensão dinâmica dos processos inerentes ao desenvolvimento dos recursos estratégicos e como estes processos contribuem para que determinadas organizações tenham um desempenho organizacional melhor que outras. Outras críticas dizem respeito à ausência de uma estrutura conceitual que demonstre o relacionamento entre recursos, embora o que se observa é que alguns recursos são desenvolvidos ou mesmo suprimidos, em função de outros recursos existentes na organização (Morecroft, 2008; Warren, 2002).

RIAE - Revista Ibero-Americana de Estratégia, São Paulo, v. 8, n. 1, p. 122-138, jan./jun. 2009. 
Visão Baseada em Recursos Dinâmicos: Estudo das Contribuições da Área de Dinâmica de Sistemas (DS) para a Teoria da Visão Baseada em Recursos (VBR)

Diante desses aspectos, faz-se necessário um corpo de conceitos que possa enriquecer a teoria da RBV, através da dinamicidade necessária para facilitar a compreensão dinâmica inerente aos processos estratégicos.

A área de Dinâmica de Sistemas (DS) estuda o comportamento dos sistemas dinâmicos, principalmente aqueles baseados em uma natureza social. Busca explicar como a estrutura de relacionamento entre variáveis no sistema define o comportamento desses sistemas, através de modelos qualitativos e quantitativos (simulações computacionais) (Morecroft \& Sterman, 1994; Sterman, 2000).

Naturalmente, esta área vêm dar uma contribuição ao aprofundamento do estudo sobre a escola RBV, tendo em vista que a estrutura de relacionamento entre variáveis e seu comportamento durante o tempo pode ser aplicado à estrutura de relacionamento entre os recursos estratégicos, suas inter-relações e influência desse sistema no desempenho da organização. Este artigo tem como objetivo avaliar as contribuições conceituais da área de Dinâmica de Sistemas (DS) para a Teoria da Visão Baseada em Recursos (VBR).

\section{TEORIA DA VISÃO BASEADA EM RECURSOS}

O princípio fundamental da RBV é que a vantagem competitiva de uma organização está relacionada diretamente ao uso e desenvolvimento de determinados recursos estratégicos disponíveis para a organização (Barney, 1991).

Dessa forma, a teoria busca esclarecer que o fato das organizações com um desempenho superior e uma vantagem competitiva alcançada não é explicado por terem uma posição produtomercado melhor ou estarem em um setor mais favorável, mas porque se apropriam de recursos que geram vantagem competitiva sustentável (Burlamaqui \& Proença, 2003). A teoria RBV, então, distingue-se da tradicional visão do posicionamento, voltando sua análise para o interior da organização e analisando forças e deficiências de seus recursos sob controle.

Barney (1991) contribuiu de maneira considerável com a teoria explicando que a vantagem competitiva deriva dos recursos e competências que uma organização controla, que necessariamente necessitam ter quatro características: precisam ser valiosos, raros, difíceis de imitar e não substituíveis.

$\mathrm{O}$ autor relata que os recursos valiosos permitem que as organizações implementem estratégias que melhorem sua eficiência e efetividade. $\mathrm{O}$ valor do recurso é proveniente de uma 
Josué Vitor de Medeiros Júnior, Miguel Eduardo Moreno Añez, Isabella Freitas G. de Vasconcelos e Fernando Porfírio S. de Oliveira

análise de mercado, e portanto da influência do recurso nas oportunidades e ameaças, e do impacto dos recursos internamente.

Recursos compartilhados por várias organizações concorrentes, por mais que tenham valor, não determinam uma vantagem estratégica sustentável. Assim, é preciso que o recurso seja raro e apenas a organização possua e controle este recurso.

Além disso, as organizações concorrentes que não conseguirem ou tiverem grande dificuldade de ter acesso a um recurso valioso e raro caracterizam o recurso como difícil de imitar. Dependência histórica, ambiguidade causal e complexidade social são fatores que, segundo Barney (1991), influenciam na dificuldade de imitação dos recursos.

Por fim, é preciso que o recurso não seja substituído por outro, pois isso possibilitaria que concorrentes tivessem acesso a recursos similares que trouxessem vantagem competitiva. Nesse contexto, o autor ressalta que uma organização terá uma vantagem competitiva sustentável quando implementar uma estratégia de criação de valor que não esteja sendo implementada simultaneamente por nenhum outro concorrente atual ou potencial e que estes concorrentes não tenham condições de replicar os benefícios dessa estratégia (Burlamaqui \& Proença, 2003).

Assim, uma organização será diferenciada em função do grupamento singular de recursos possuído por ela, sejam os ativos organizacionais mais conhecidos, sejam competências ou capacidades (Fleury \& Fleury, 2003).

Deve-se destacar ainda recentes contribuições dadas à teoria VBR. Lavie (2006) apresenta uma extensão da teoria para organizações interconectadas, redefinindo atributos relacionados ao grau de compartilhamento entre os recursos, e ressaltando que em alianças empresariais, os relacionamentos entre os recursos de empresas distintas são tão importantes quanto os próprios recursos.

Dada a complexidade natural na gestão de recursos intangíveis, como aqueles relacionados às pessoas, a área intitulada strategic human resource management estende a teoria, reconhecendo que aspectos relacionados aos recursos humanos, como habilidades, interações e comportamentos dos funcionários, influenciam diretamente na concepção e implementação das estratégias organizacionais (Colbert, 2004).

Outros estudos enfatizam o desenvolvimento de estratégias organizacionais baseado em recursos naturais e na responsabilidade ambiental dessas organizações (Hart, 1995).

Barney, Wright e Ketchen Junior (2001) mostram como diversas contribuições da VBR têm influenciado e sido influenciadas por outras áreas, como empreendedorismo, através de pesquisa visando compreender, por exemplo, como os empreendedores se envolvem no processo de

RIAE - Revista Ibero-Americana de Estratégia, São Paulo, v. 8, n. 1, p. 122-138, jan./jun. 2009. 
Visão Baseada em Recursos Dinâmicos: Estudo das Contribuições da Área de Dinâmica de Sistemas (DS) para a Teoria da Visão Baseada em Recursos (VBR)

compreensão, descoberta e identificação de oportunidades; na área de marketing, demonstrando a importância nos recursos de mercado e da empresa para criação e sustentação de valor para o cliente, além de abordagens como capacidades dinâmicas e relacionamentos entre governança corporativa e a VBR.

\section{DINÂMICA DE SISTEMAS}

Decisões e aprendizado em um mundo que apresenta crescente complexidade dinâmica exigem que os profissionais sejam pensadores sistêmicos. Isso significa que há necessidade de uma expansão nas fronteiras dos modelos mentais e desenvolvimento de ferramentas para compreender como a estrutura de sistemas complexos afeta o comportamento do próprio sistema (Morecroft \& Sterman, 1994).

Dessa forma, gestores estão envolvidos muitas vezes em uma complexidade dinâmica (Senge, 1990) em que situações organizacionais supostamente previsíveis produzem resultados surpreendentes ou até opostos aos desejados. O êxito, na maioria das situações gerenciais, está na compreensão de situações de complexidade dinâmica que, embora sejam comuns nos ambientes organizacionais, são pouco estudadas nos cursos de Administração.

A Dinâmica de Sistemas (DS) é um corpo de teorias e um conjunto de ferramentas conceituais que auxiliam na compreensão da estrutura e na dinâmica de sistemas complexos. DS também é um método rigoroso de modelagem que utiliza simulações em computador para definir organizações e políticas mais efetivas (Sousa, 2006).

Tais ferramentas permitem a criação de simuladores gerenciais (Sterman, 2000) - mundos virtuais onde espaço e tempo podem ser comprimidos e desacelerados de tal forma a permitir a experimentação de efeitos colaterais de longo prazo, aprendizado e o projeto de estruturas e estratégias de alto desempenho.

A Dinâmica de Sistemas foi concebida há 45 anos quando Jay Forrester publicou o livro Industrial Dynamics. Desde então, este campo tem se expandido passando a contemplar pesquisadores e praticantes das mais diversas áreas do conhecimento, como medicina, economia, sociologia, planejamento militar, além do domínio dos negócios (Fernandes, 2001).

Como o nome sugere, a Dinâmica de Sistemas busca entender a evolução de um sistema ao longo do tempo. Essa abordagem tem como principal premissa o fato de que o comportamento de um sistema é determinado por sua estrutura interna. Logo, utilizando-se de uma linguagem própria 
Josué Vitor de Medeiros Júnior, Miguel Eduardo Moreno Añez, Isabella Freitas G. de Vasconcelos e Fernando Porfírio S. de Oliveira

para modelar um sistema, é possível investigar o seu comportamento ao longo do tempo, ou seja, testar os diferentes tipos de comportamento que o sistema real pode experimentar, tornando viável a identificação e a avaliação de melhorias potenciais, através da adoção de um ou mais pontos de alavancagem (Fernandes, 2001).

DS é uma metodologia que busca mapear sistemas organizacionais ou sociais, procurando examinar a inter-relação de suas influências, vendo-as num contexto sistêmico e entendendo-as como parte de um processo comum. Por intermédio da simulação, propõe a compreensão de como o sistema em foco evolui no tempo e como mudanças em suas partes afetam todo o seu comportamento (Andrade, 1997).

De acordo com a perspectiva sistêmica, da qual a DS é derivada, a maioria dos gestores procura resolver os problemas organizacionais de maneira reativa e focada em eventos e soluções de curto prazo. Comumente eles se baseiam em conhecimento e experiências anteriores e analisam o problema dividindo-o em várias partes. No entanto, a forma mais profunda de resolução de problemas é a identificação das causas subjacentes aos padrões de comportamento do sistema, permitindo que esses padrões sejam modificados através da compreensão estrutural do sistema (Senge, 1990). Essa abordagem deve considerar toda a estrutura sistêmica, as partes do sistema e seus inter-relacionamentos.

Segundo Senge (1990), a prática de operar com base nos eventos imediatos e superficiais são formas limitadas no sentido de produzir resultados sustentáveis e, não raro, trazem consequências contrárias àquelas desejadas.

A fim de tratar a complexidade na prática, a DS busca construir modelos representativos do sistema, simulando seu comportamento ao longo do tempo, reproduzindo comportamentos problemáticos e avaliando o impacto de novas políticas na condução desse sistema.

Diferente dos sistemas lineares, em um sistema dinâmico as decisões são derivadas de informações sobre o sistema. Essas decisões são convertidas em ações que interferem no comportamento do sistema. Quando novas informações são geradas, é possível avaliar qual o impacto da decisão passada no sistema em questão (Fernandes, 2003). A modelagem da DS está orientada para mapear a estrutura e, por intermédio da simulação, verificar o impacto das decisões e testar diferentes políticas e soluções para operação do sistema. Tais procedimentos abrem espaço para diferentes tipos de aplicações da DS no âmbito da gestão.

A DS se utiliza de ferramentas de simulação e notadamente de uma linguagem intitulada fluxos e estoques para representar contextos complexos e sistêmicos, que podem ser simulados através de softwares específicos para tal fim (Sterman, 2000).

RIAE - Revista Ibero-Americana de Estratégia, São Paulo, v. 8, n. 1, p. 122-138, jan./jun. 2009. 
Visão Baseada em Recursos Dinâmicos: Estudo das Contribuições da Área de Dinâmica de Sistemas (DS) para a Teoria da Visão Baseada em Recursos (VBR)

Um exemplo de modelo quantitativo de fluxos e estoques simples é demonstrado na Figura 1.

Figura 1 - Exemplo de um Diagrama de Fluxos e Estoques

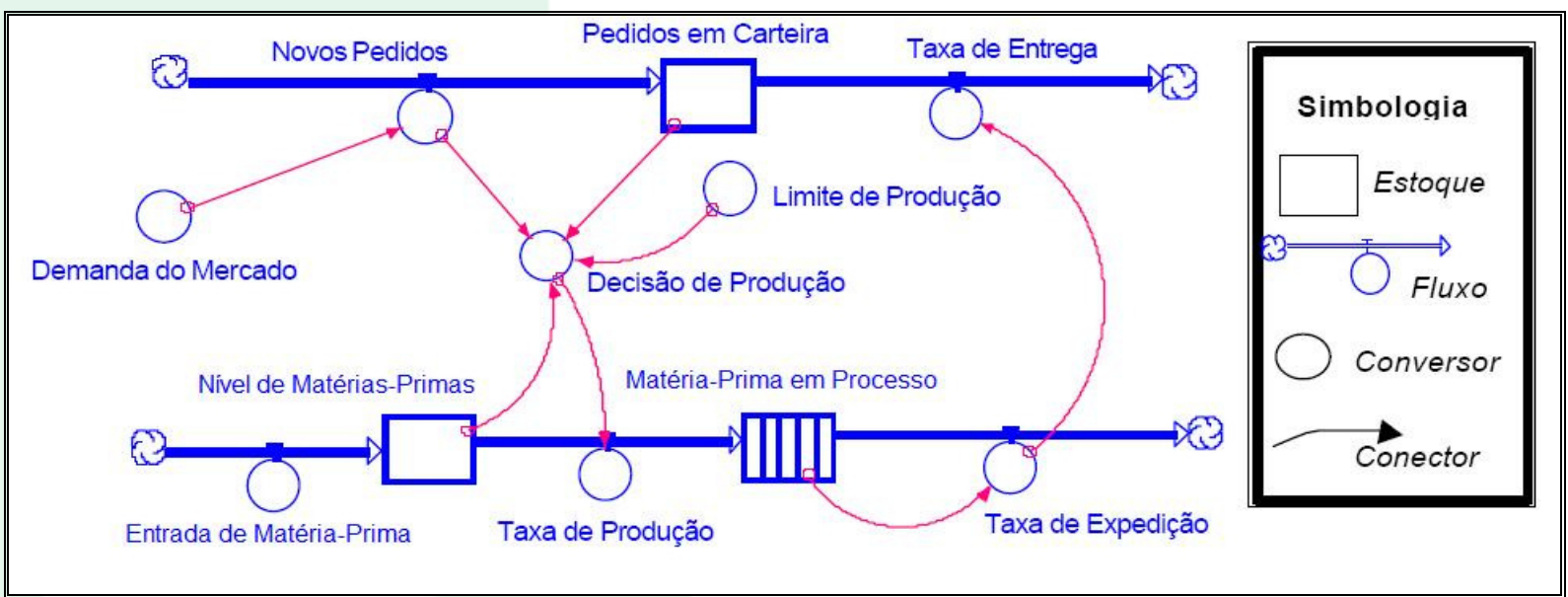

Fonte: Fernandes (2001)

Conforme demonstrado na Figura 1, os estoques representam acúmulos de materiais ou informações que caracterizam o estado do sistema. Dessa forma, fornecem aos gestores informações nas quais podem se basear para a tomada de decisões. Eles são reconhecidos em um sistema porque permanecem constantes quando todas as atividades cessam, provendo memória e inércia ao sistema. São responsáveis ainda, pelos atrasos existentes no sistema, decorrentes do acúmulo de informações originado pela diferença entre o fluxo de entrada de um processo e o fluxo de saída. Um dos estoques representados na Figura 1 é intitulado Pedidos em Carteira e representa a quantidade de pedidos acumulados dos clientes pela organização.

Por outro lado, os fluxos caracterizam a dinâmica do sistema e representam as atividades que produzem crescimento ou redução dos estoques, sendo dependentes destes. As atividades de Novos Pedidos, que incrementam a quantidade de pedidos em carteira, e a Taxa de Entrega, que diminui este nível, são os fluxos do sistema. Caso as atividades cessem ou se mantenham com valores iguais, não haverá nenhuma alteração nos pedidos em carteira.

RIAE - Revista Ibero-Americana de Estratégia, São Paulo, v. 8, n. 1, p. 122-138, jan./jun. 2009. 


\section{CONTRIBUiÇÃo DA DINÂMICA DE SISTEMAS PARA VISÃo BASEADA EM RECURSOS}

Muitos autores veem a necessidade da incorporação de aspectos dinâmicos relacionados ao processo de desenvolvimento de estratégias para teorias como a RBV. Porter (1991, citado por Gary, Kunc, Morecroft \& Rockart, 2008), por exemplo, reconhece que embora existam disponíveis na literatura acadêmica e comercial diversos frameworks conceituais que explicam o sucesso competitivo de uma organização em um determinado ponto no tempo específico, o entendimento sobre o processo dinâmico em que as organizações desenvolvem suas estratégias não foi explorado e nem está claro em tais publicações.

Dierickx e Cool (1989) apresentaram uma visão de natureza dinâmica do processo de elaboração e execução de estratégias, descrevendo a dinâmica de recursos nas organizações como fluxos e estoques que geram acúmulos de ativos organizacionais. Estes autores ressaltam que os gestores realizam uma série de decisões que influenciam na evolução da dinâmica dos recursos e nas posições competitivas das organizações, favorecendo a heterogeneidade entre as organizações ao longo do tempo.

Esse interesse pela heterogeneidade nos desempenhos organizacionais pela comunidade científica da área de estratégia torna necessários trabalhos que busquem evidências entre os padrões de decisões dos gestores e os padrões de trajetórias do desempenho ao longo do tempo (Gary et al., 2008).

A grande questão a ser estudada e aprofundada por estes e demais autores é buscar compreender por que determinadas organizações apresentam desempenho superior enquanto outras não, dentro de uma perspectiva dinâmica. A Figura 2 a exibe esta postura, ressaltando a necessidade para se compreender como, por que e onde o desempenho organizacional tem se desenvolvido ao longo do tempo e que possibilidades podem ocorrer no futuro se determinadas decisões forem tomadas, com o objetivo de gerar aprendizado.

RIAE - Revista Ibero-Americana de Estratégia, São Paulo, v. 8, n. 1, p. 122-138, jan./jun. 2009. 
Visão Baseada em Recursos Dinâmicos: Estudo das Contribuições da Área de Dinâmica de Sistemas (DS) para a Teoria da Visão Baseada em Recursos (VBR)

Figura 2 - Dinâmica no desempenho organizacional

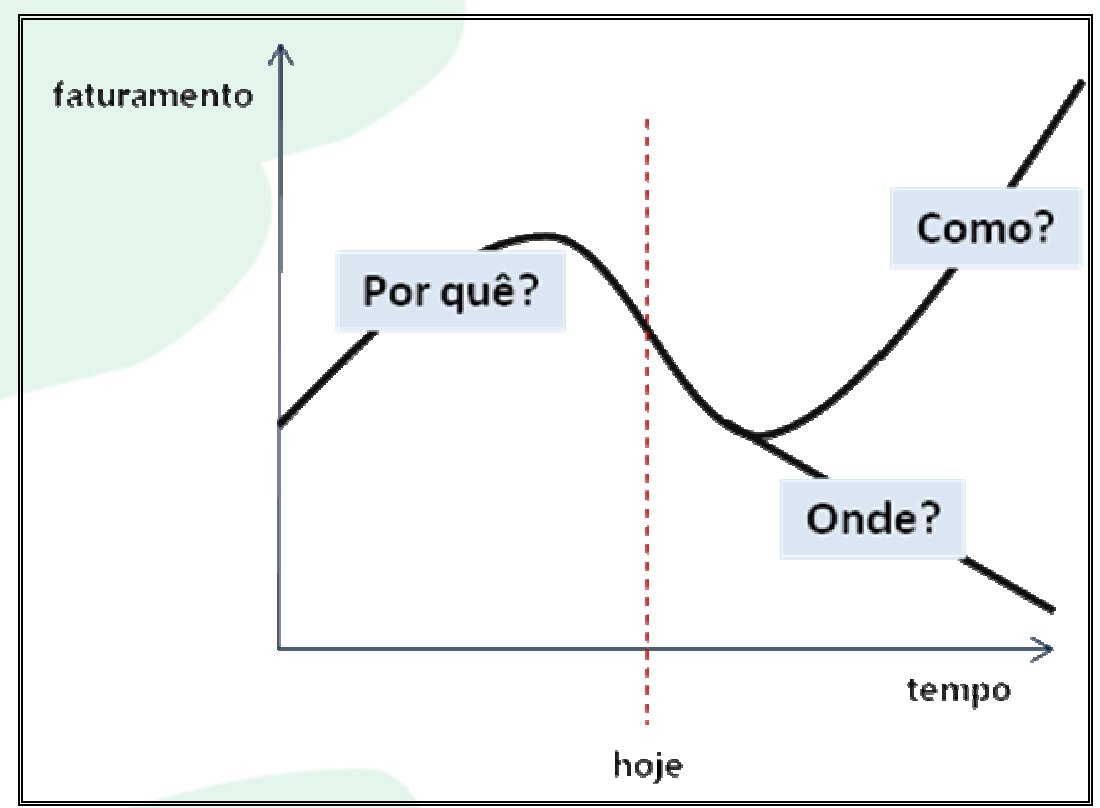

Fonte: Adaptado de Warren (2002)

A Figura demonstra perspectivas passadas e futuras que precisam ser analisadas. Assim, fazse necessário compreender por que o desempenho organizacional de uma empresa, medido na figura pelo faturamento, tem seguido um determinado padrão histórico ao longo do tempo, para que se possa compreender que recursos influenciaram neste desempenho, a partir de decisões tomadas anteriormente.

Além disso, em relação ao futuro, deve-se avaliar para onde o desempenho da empresa levará a todos, caso se mantenham as condições atuais. Analisar esses aspectos e compreender como se pode alterar padrões no futuro permite que se aprenda a partir das análises realizadas.

Nesse contexto, a Dinâmica de Sistema provê ferramentas quantitativas de simulação para que modelos possam ser construídos e questionamentos como estes possam ser realizados, tendo como base os recursos tangíveis e intangíveis da organização, seus inter-relacionamentos e padrões de comportamento ao longo do tempo.

Assim, a DS pode contribuir com a teoria RBV, através de suas ferramentas e conceitos, como fluxos e estoques, usados para representar modelos de simulação e enlaces de feedbacks, na compreensão da não-linearidade dos sistemas de complexidade dinâmica, como aqueles da área de estratégia organizacional (Morecroft \& Sterman, 1994; Sterman, 2000).

Ford e Mahieu (1998) elaboraram um framework para operacionalizar modelos que pudessem ser adotados para se construir e analisar estratégias, baseados na VBR. Os autores 
Josué Vitor de Medeiros Júnior, Miguel Eduardo Moreno Añez, Isabella Freitas G. de Vasconcelos e Fernando Porfírio S. de Oliveira

constataram que uma das principais causas para dificuldade em se operacionalizar ideias baseadas no RBV é seu alto nível de abstração. Assim, ao operacionalizar a VBR houve a possibilidade de se formalizar ideias e conceitos em modelos práticos que podem, inclusive, ser simulados, facilitando a formulação, análise de estratégias e decisões.

O método proposto por esses autores parte da identificação dos recursos tangíveis e intangíveis principais de uma organização; levantamento de como esses recursos têm se comportado ao longo do tempo, através de gráficos; elaboração de um diagrama causal (de natureza qualitativa), para se compreender como os recursos de relacionam entre si; elaboração de políticas e planos estratégicos a partir da análise do diagrama, possibilitando que cenários possam ser construídos; e, por fim, desenvolvimento de um modelo de simulação sistêmico, através da linguagem de fluxos e estoques, permitindo que os gestores possam experimentar decisões em um framework dentro da realidade estratégica da organização.

Um outro framework conceitual vêm sendo desenvolvido e utilizado em formulações de estratégias de diversas organizações por Warren (2002), conhecido como Strategy Dynamics, em que foram incorporados conceitos da DS para a teoria RBV, explorando a dinamicidade inerente ao processo de desenvolvimento de estratégias em organizações e como seus recursos se desenvolvem ao longo do tempo.

O autor descreve a existência dos ciclos de reforços e equilíbrios ao qual o sistema organizacional está envolvido dentro de uma perspectiva estratégica, na importância dos recursos intangíveis e sua influência nos recursos tangíveis que demonstram o desempenho da organização, em como determinados recursos são influenciados ou influenciam outros e seus padrões de desenvolvimento ao longo do tempo e nas dinâmicas relacionadas à rivalidade entre organizações concorrentes, em que os mesmos recursos são conquistados por concorrentes distintos (Warren, 2002, 2008).

Esse autor elaborou então uma série de passos para o desenvolvimento de uma arquitetura estratégica que visa otimizar o desempenho organizacional no apoio à gestão estratégica:

- Identificar o desenvolvimento histórico do desempenho organizacional - Para que haja maior compreensão sobre como o desempenho organizacional tem se comportado dinamicamente é necessário definir que medidas, como volume de vendas, clientes, etc., serão adotadas, qual o horizonte de tempo e escalas serão considerados, além de levantar esses valores em um ou vários gráficos;

RIAE - Revista Ibero-Americana de Estratégia, São Paulo, v. 8, n. 1, p. 122-138, jan./jun. 2009. 
Visão Baseada em Recursos Dinâmicos: Estudo das Contribuições da Área de Dinâmica de Sistemas (DS) para a Teoria da Visão Baseada em Recursos (VBR)

- Identificar os principais recursos estratégicos - Faz-se necessário ainda se identificar exatamente quais os recursos mais importantes para a organização, ou seja, aqueles recursos responsáveis pela vantagem competitiva sustentável, sejam tangíveis (clientes, faturamento, funcionários, etc.) ou intangíveis (como habilidades, imagem da organização, etc.);

- Identificar e quantificar os fluxos responsáveis pelas alterações no padrão de acumulação dos recursos - Nesta etapa, será necessário quantificar as alterações no comportamento dos recursos levantados. Isso significa definir quantitativamente que fórmulas matemáticas definem os fluxos de entrada e saída dos recursos, responsáveis por seu desenvolvimento ou declínio. Para facilitar a compreensão, um recurso é calculado em função de suas entradas menos suas saídas. Os clientes existentes atualmente na empresa (um recurso possivelmente estratégico) são calculados em função de todos os clientes que passaram a esta condição menos todos aqueles que deixaram de ser clientes da empresa;

- Identificar as dependências entre os recursos e nos seus fluxos - Uma vez definidos os padrões de comportamento de crescimento e declínio dos recursos, faz-se necessário definir os inter-relacionamentos entre eles, isso permite que sejam identificados relações causais entre os recursos;

- Combinar as interdependências entre os fluxos e recursos em uma visão unificada da arquitetura estratégica da organização - faz-se necessário então avaliar que relacionamentos ou variáveis adicionais podem ser necessários para finalizar a arquitetura estratégica da organização e identificar possíveis erros ou discrepâncias;

- Executar a arquitetura resultante através de simulações - Uma vez finalizada a arquitetura estratégica em uma linguagem de fluxos e estoques apropriada fornecida pela DS, pode-se realizar simulações e decisões para avaliar como os recursos se relacionam e qual o comportamento destes ao longo do tempo;

- Analisar e revisar as políticas e compreender como a arquitetura atual influencia no desempenho organizacional - Normalmente, simulações podem gerar novas ideias a cerca da estratégia organizacional, possibilitando que políticas podem ser elaboradas ou adicionadas.

RIAE - Revista Ibero-Americana de Estratégia, São Paulo, v. 8, n. 1, p. 122-138, jan./jun. 2009. 
Josué Vitor de Medeiros Júnior, Miguel Eduardo Moreno Añez, Isabella Freitas G. de Vasconcelos e Fernando Porfírio S. de Oliveira

A Figura 3 exibe uma parte da arquitetura estratégica de uma companhia aérea, sendo os seus recursos estratégicos - como funcionários, passageiros potenciais ou ativos, aviões, etc. representados por caixas e fluxos que entram e saem das caixas responsáveis pelo acúmulo e diminuição nos níveis dos recursos.

Outras variáveis ainda foram inseridas no modelo (como qualidade do pessoal, custos, etc.) para que este possa representar a estratégia da organização.

Figura 3 - Parte da arquitetura estratégica de uma companhia aérea

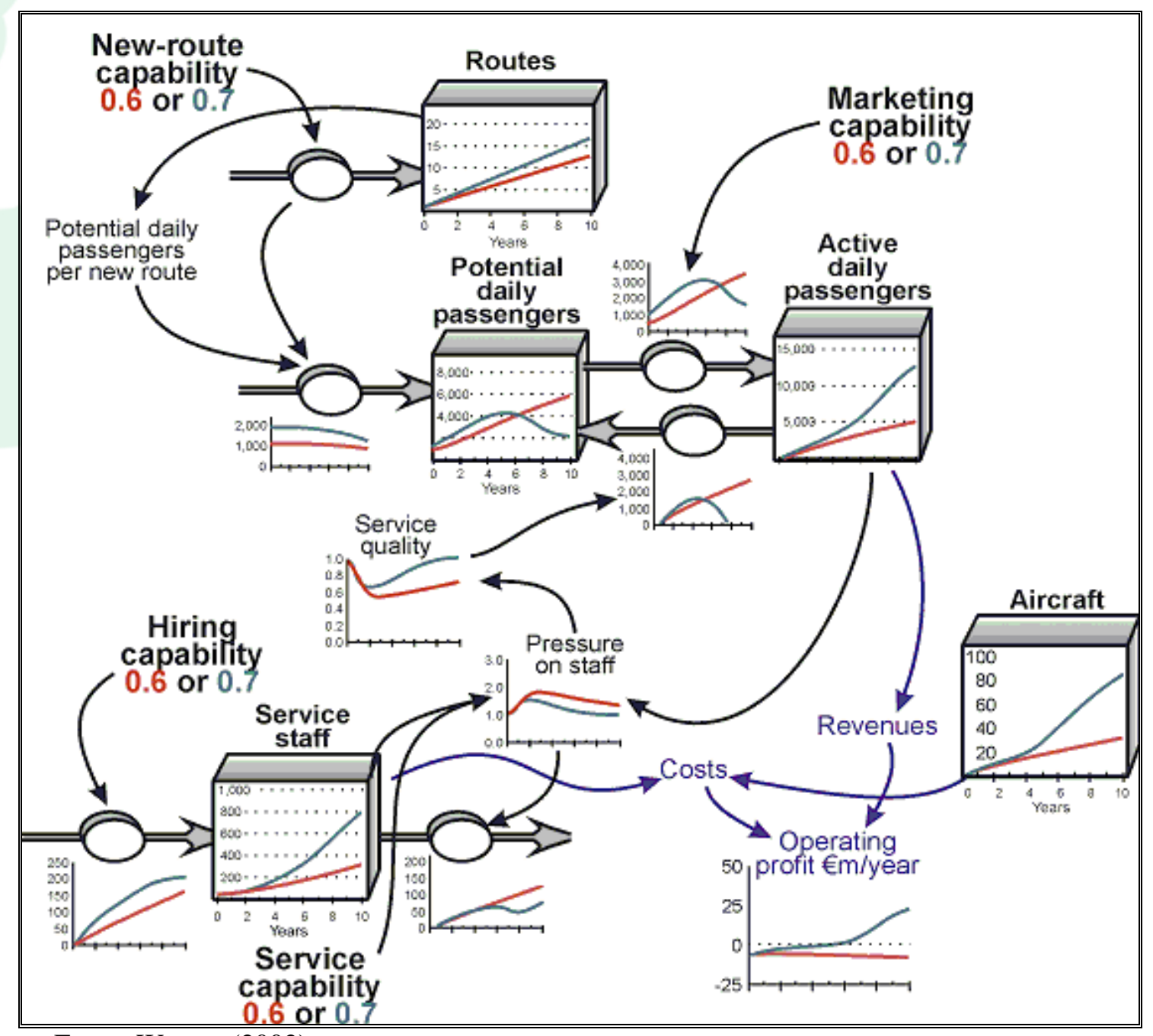

Fonte: Warren (2002)

\section{CONCLUSÕES}

RIAE - Revista Ibero-Americana de Estratégia, São Paulo, v. 8, n. 1, p. 122-138, jan./jun. 2009. 
Visão Baseada em Recursos Dinâmicos: Estudo das Contribuições da Área de Dinâmica de Sistemas (DS) para a Teoria da Visão Baseada em Recursos (VBR)

Diante do levantamento bibliográfico realizado, pode-se constatar a importância da contribuição da Dinâmica de Sistemas para a teoria da Visão Baseada em Recursos, tendo em vista que enquanto esta tem se desenvolvido e se tornado de grande importância como modelo para que as organizações desenvolvam suas estratégias, vislumbrando a importância de seus recursos estratégicos para vantagem competitiva sustentável, aquela pode incorporar aspectos mais representativos no sentido de permitir que os modelos estratégicos desenvolvidos possam ser avaliados e experimentados.

Assim, os gestores podem construir tais modelos e tomar decisões, visualizando como os recursos se relacionam e influenciam no desempenho da organização, sem que necessariamente estejam expostos aos riscos financeiros da realidade.

Dessa forma, caminha-se para que as estratégias possam ser construídas e testadas, enriquecendo ainda mais o processo, favorecendo o compartilhamento de modelos mentais entre os gestores (ou seja, suas visões de mundo) e permitindo que a comunicação seja mais efetivas.

Enquanto perspectivas de trabalhos futuros, pode-se vislumbrar a aplicação desses conceitos no ensino de disciplinas de estratégias, para que os alunos possam ter uma participação ativa no processo de ensino-aprendizagem e compreendam como suas decisões e construção de estratégias impactam no sistema organização. Assim, esta perspectiva também poderá contribuir na formação dos alunos da área de administração, provendo verdadeiros laboratórios de gestão, através de diversos modelos de simulação construídos a partir de estudos de casos.

\section{REFERÊNCIAS}

Andrade, A. L. (1997). Pensamento sistêmico: um roteiro básico para perceber as estruturas da realidade organizacional. Revista Eletrônica de Administração, 3(1), 1-30.

Barney, J. (1991). Firm resources and sustained competitive advantage. Journal of Management, 17(1), 99-120.

Barney, J., Wright, M., \& Ketchen Jr. (2001). The resource-based view of the firm: ten years after 1991. Journal of Management, 27(6), 625-641.

Burlamaqui, L., \& Proença, A. (2003). Inovação, recursos e comprometimento: em direção a uma teoria estratégica da firma. Revista Brasileira de Inovação, 2(1), 79-110.

Colbert, B. A. (2004). The complex resource-based view: implications for theory and practice in strategic human resource management. Academy of Management Review, 29(3), 341-358.

RIAE - Revista Ibero-Americana de Estratégia, São Paulo, v. 8, n. 1, p. 122-138, jan./jun. 2009. 
Josué Vitor de Medeiros Júnior, Miguel Eduardo Moreno Añez, Isabella Freitas G. de Vasconcelos e Fernando Porfírio S. de Oliveira

Dierickx, I., \& Cool, K. (1989). Asset stock accumulation and sustainability of competitive advantage. Management Science, 33(12), 1504-1511.

Fernandes, A. C. (2003). Scorecard dinâmico: em direção à integração da dinâmica de sistemas com o balanced scorecard. Tese de Doutorado, Programa de Engenharia de Produção, Universidade Federal do Rio de Janeiro, Rio de Janeiro.

Fernandes, A. C. (2001). Dinâmica de sistemas e business dynamics: tratando a complexidade em ambiente de negócios [CD-ROM]. In Anais do Encontro Nacional de Engenharia de Produção, 21. Salvador: Abepro.

Fleury, A. C. C., \& Fleury, M. T. L. (2003). Estratégias competitivas e competências essenciais: perspectivas para a internacionalização da indústria no Brasil. Gestão \& Produção, 10(2), 129-144.

Ford, D. N., \& Mahieu, L. A. (1998). Operationalising the resource-based view of the firm [CDROM]. In Proceedings of International Conference of the System Dynamics Society, 16. Albany: The System Dynamics Society.

Gary, M. S., Kunc, M. H., Morecroft, J., \& Rockart, S. (2008). System dynamics and strategy. System Dynamics Review, 24(4), 407-429.

Hart, S. (1995). A natural-resource-based view of the firm. Academy of Management Review, 20(4), 986-1014.

Lavie, D. (2006). The competitive advantage of interconnected firms: an extension of the resourcebased view. Academy of Management Review, 31(3), 638-658.

Mintzberg, H., Ahlstrand, B., \& Lampel, J. (2000). Safári de estratégia: um roteiro pela selva do planejamento estratégico (N. Montingelli Jr., Trad.). Porto Alegre: Bookman.

Morecroft, J. D. W. (2008). System dynamics, RBV and behavioural theories of firm performance: lessons from people express. Recuperado em 17 outubro, 2008, de http://www.systemdynamics.org/conferences/2008/proceed/papers/MOREC159.pdf

Morecroft, J. D. W., \& Sterman, J. D. (1994). Modeling for learning organizations. Portland: Productivity Press.

Senge, P. M. (1990). A quinta disciplina: arte, teoria e prática da organização de aprendizagem. São Paulo: Best Seller.

Sheehan, N. T. (2006). Understanding how resources and capabilities affect performance: actively applying the resource-based view in the classroom. Journal of Management Education, 30(3), 421430.

Sousa, G. W. L. (n.d.). System dynamics. Recuperado em 07 de junho, 2006, de http://www.numa.org.br/conhecimentos/conhecimentos_port/pag_conhec/System\%20Dynamics.ht ml\#instrucao

Sterman, J. (2000). Business dynamics: systems thinking and modelling for a complex world. Boston: Irwin McGraw-Hill.

RIAE - Revista Ibero-Americana de Estratégia, São Paulo, v. 8, n. 1, p. 122-138, jan./jun. 2009. 
Visão Baseada em Recursos Dinâmicos: Estudo das Contribuições da Área de Dinâmica de Sistemas (DS) para a Teoria da Visão Baseada em Recursos (VBR)

Vasconcelos, F. C., \& Cyrino, A. B. (2000). Vantagem competitiva: os modelos teóricos atuais e a convergência entre estratégia e teoria organizacional. Revista de Administração de Empresas, 40(4), 20-37.

Warren, K. (2002). Competitive strategy dynamics. Chichester: John Wiley \& Sons.

Warren, K. (2008). Strategic management dynamics. Chichester: John Wiley \& Sons.

\section{Recebido: 15/04/2009}

Aprovado: 08/06/2009

RIAE - Revista Ibero-Americana de Estratégia, São Paulo, v. 8, n. 1, p. 122-138, jan./jun. 2009. 